\title{
Frequency Detectors for PLL Acquisition in Timing and Carrier Recovery
}

\author{
DAVID G. MESSERSCHMITT, SENIOR MEMBER. IEEE
}

\begin{abstract}
A significant problem in phase-locked loop (PLL) timing and carrier extraction is the initial acquisition. Very narrow loop bandwidths are generally required to control phase jitter, and acquisition may depend on an extremely accurate initial VCO frequency (VCXO) or sweeping. We describe two simply implemented frequency detectors which, when added to the traditional phase detector, can effect acquisition even with very small loop bandwidths and large initial frequency offsets.

The first is the quadricorrelator, previously applied to timing recovery by Bellisio, while the second is new, and called a rotational frequency detector. The latter, while limited to lower frequencies and higher signal-to-noise ratios, is suitable for many applications and can be implemented with simpler circuitry.
\end{abstract}

\subsection{INTRODUCTION}

$\mathbf{T}$ HE initial acquisition of a phase-locked loop (PLL) when used for timing or carrier extraction is a significant practical problem, since the narrow loop bandwidth generally required for jitter requirements severely restricts the pull-in range. Methods widely employed to effect acquisition include [1]

a) compromises in loop filter design,

b) highly accurate initial VCO frequency (VCXO),

c) sweeping of the $\mathrm{VCO}$, and

d) in-lock detection with switching of loop filter.

In many instances, as in carrier recovery, several of these methods may be simultaneously employed.

There is a fifth method of effecting acquisition [1], which seems to have been first suggested by Richman [2] , and that is to add a frequency detector (FD) to the traditional PLL phase detector (PD) in the manner of Figure 1. With a large initial VCO frequency offset, the PD output has essentially a zero d.c. output, and the FD generates a voltage proportional to the frequency difference between input and VCO, driving that difference to zero. The PD takes over when the frequency difference is small, completing the acquisition. When the PLL is in-lock, the FD output will have at the least zero mean, and optimistically will be identically zero, automatically allowing the PD and its loop filter to govern the loop dynamics. The beauty of this approach is that a crystal controlled VCO (VCXO) can often be exchanged for the additional FD circuitry in timing recovery applications, an advantageous tradeoff in this age of integrated circuitry. In carrier recover, a VCXO is

Paper approved by the Editor for Data Communication Systems of the IEEE Communications Society for publication without oral presentation. Manuscript received October 23, 1978; revised May 4, 1979. This research was performed for the VIDAR Division of TRW, Mountain View, CA.

The author is with the Department of Electrical Engineering and Computer Science, University of California, Berkeley, CA 94720.

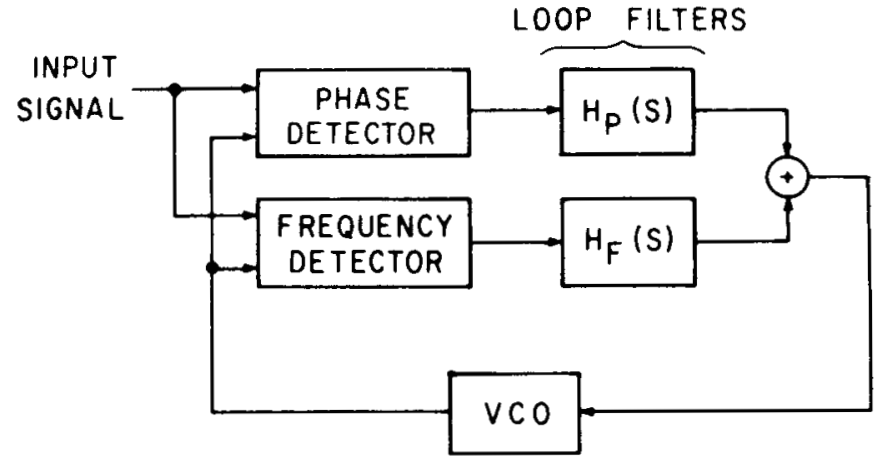

Fig. 1. PLL with Phase and Frequency Detector.

often still required because of the problem of false locking to a data sideband, but the sometimes troublesome in-lock detector and/or sweeping circuitry can be eliminated and the PD loop filter can be designed virtually independently of acquisition considerations, removing a significant burden from the designer.

This paper will discuss two specific FD's, each of which is applicable to both timing and carrier recovery. The first is the quadricorrelator described by Richman [2], which was more recently rediscovered by Pickard [3-5], Bellisio [6], and in modified form by Park [7], Cahn [8] and Citta [14]. These authors have discussed its applicability to sinusoid $[2,3]$ and narrowband Gaussian process [2-4] input signals, to timing recovery [6], and to Costas loop carrier recovery for biphase modulation [8]. We will show here that the quadricorrelator is more generally applicable to carrier recovery for any modulation method which has a power spectrum symmetrical about the carrier frequency. This includes most data modulation methods, with the notable exceptions of single and vestigal sideband modulation.

The second FD, called a rotational FD, is new, and unlike the quadricorrelator is implemented with predominately digital circuitry. As a consequence, its operation is limited to lower frequencies, but where applicable it is more amenable to integrated circuitry realization because of the elimination of multipliers and filtering functions. Its operation depends on detecting, with simple circuitry, the direction of rotation of the signal constellation.

For completeness we mention the papers by Oberst [9], describing an FD for two square waves (useful in frequency synthesis *), and Runge [10], describing an unrelated FD for timing and carrier recovery applications.

\footnotetext{
* The FD's described here can be used for two sinusoids or square waves, but appear to have greater complexity than Oberst's circuits.
} 


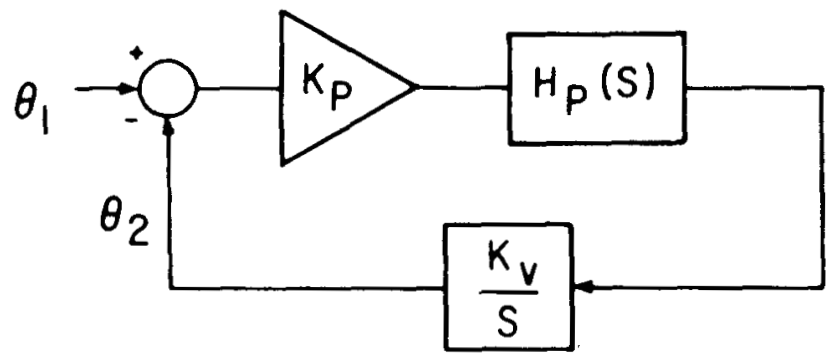

(a)

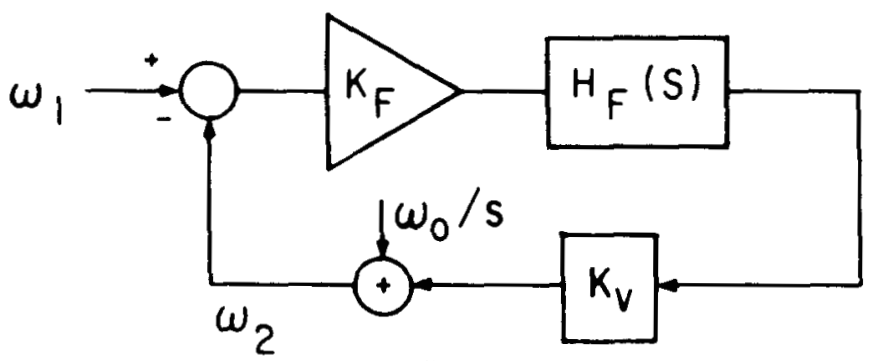

(b)

Fig. 2. Linearized Loop Models.

While the primary purpose of this paper is to describe and analyze the FD techniques, we first discuss in Section 2.0 the choice of loop filters. Then in Section 3.0 we focus on the quadricorrelator and rotational FD, and describe experimental results in Section 4.0 .

\subsection{LOOP FILTERS}

Assuming the input signal to the PLL of Figure 1 is of the form $\sin \left(\omega_{1} t+\theta_{1}\right)$, the VCO output is $\cos \left(\omega_{2} t+\theta_{2}\right)$, and the $\mathrm{PD}$ and FD are both linear, the linearized models of Figure 2 result. The phase-locked loop of Figure 2(a) governs after lock has been achieved, while the frequency locked loop of Figure 2(b) governs the acquisition behavior. The design parameters are the PD and FD constant $K_{p}$ and $K_{f}$, the VCO constant $K_{v}$, and the VCO free-running frequency $\omega_{0}$.

The loop dynamics are governed by the standard closed loop phase transfer function

$$
\frac{\theta_{2}(s)}{\theta_{1}(s)}=\frac{K_{p} K_{v} H_{p}(s)}{s+K_{p} K_{v} H_{p}(s)}
$$

plus a transfer function governing acquistion

$$
\omega_{1}(s)-\omega_{2}(s)=\frac{\omega_{1}(s)-\omega_{0} / s}{1+K_{f} K_{v} H_{f}(s)}
$$

Bellisio [6] recommends a proportional plus integral PD loop filter,

$$
H_{p}(s)=\mu_{1}+\frac{\mu_{2}}{s}
$$

which is a good choice since the static phase error is small [1] and the usual concern with the integrator being initially

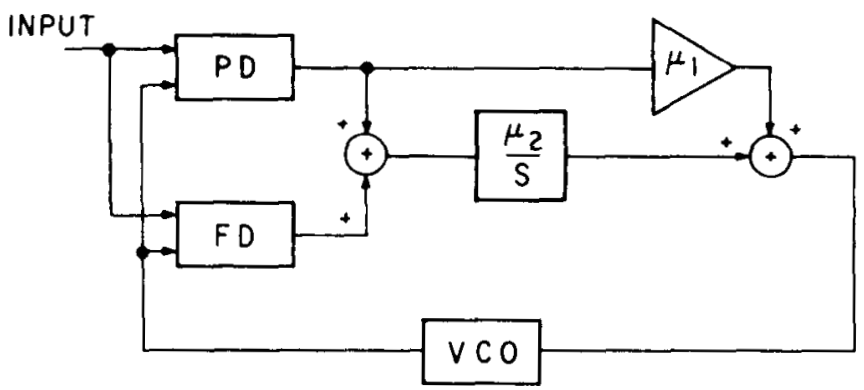

Fig. 3. Choice of Loop Filters.

saturated is alleviated due to the action of the FD. He also recommends that the $\mathrm{FD}$ use the same loop filter (that is, the summer in Figure 1 be placed in front of a single loop filter of type (2.3)). This latter choice is shown to be disadvantageous when we calculate the time response due to a step frequency change $\omega_{1}(s)=\omega_{1} / s$ from $(2.2)$,

$$
\omega_{1}(t)-\omega_{2}(t)=\frac{\omega_{1}-\omega_{0}}{1+\mu_{1} K_{f} K_{v}} e^{-t / \tau}
$$

where the time constant is

$$
\tau=\frac{\mu_{1}}{\mu_{2}}+\frac{1}{\mu_{2} K_{f} K_{v}} .
$$

Thus, we see that, as expected, fastest acquisition occurs for $K_{f}$ large, but the time constant is limited to $\tau=\mu_{1} / \mu_{2}$. Physically, this limitation on speed of acquisition is due to the proportional part of the filter, which initially reduces the frequency error and slows the charging of the integrator. The solution is to eliminate the proportional filter

$$
H_{f}(s)=\frac{\mu_{2}}{s}
$$

resulting in the configuration of Figure 3 . The FD charges the integrator capacitor to the correct voltage to reduce the frequency error to zero (in spite of any initial saturation), and inlock the PD maintains that charge. While (2.4) predicts that increasing $K_{f}$ can result in arbitrarily fast acquisition, in practice the fact that the FD output will have a randomly fluctuating voltage on its output in-lock places a practical limit on the size of $K_{f}$.

\subsection{SPECIFIC FD DESIGNS}

\subsection{Quadricorrelator Frequency Detector}

The quadricorrelator, as shown in Figure 4, consists of two quadrature mixers, a differentiator in the in-phase channel, and a cross-correlator.** The mean value of $p(t)$ is proportional to the difference between the center frequency of the power spectrum of $r(t)$ and $\omega_{2}$. While this property has been demonstrated for sinusoidal $[2,3]$ and Gaussian [3-5] inputs $r(t)$, it can be easily established in general. In particular, if

** The similarity of the quadricorrelator to the PD of a Costas loop [11] is striking. 


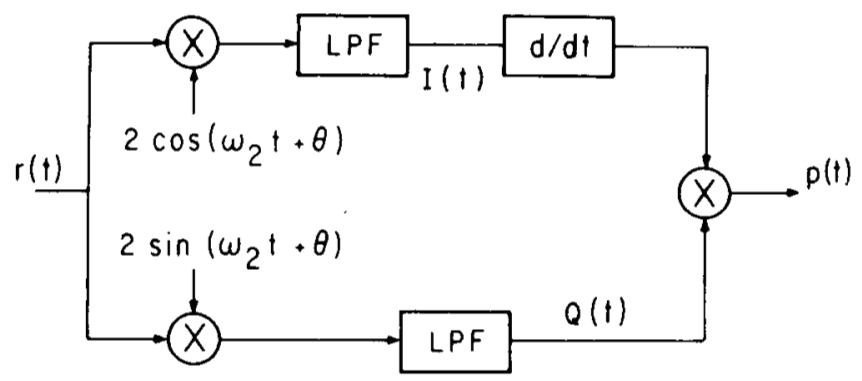

Fig. 4. Quadricorrelator.

$r(t)$ has a power spectrum symmetric about the radian frequency $\omega_{1}$, it can be expanded in the form

$$
r(t)=x_{c}(t) \cos \omega_{1} t-x_{s}(t) \sin \omega_{1} t
$$

where $x_{c}(t)$ and $x_{s}(t)$ are uncorrelated. It is shown in Appen$\operatorname{dix} A$, using (3.1), that

$$
E p(t)=\Delta \omega \sigma_{r}^{2}
$$

where $\Delta \omega$ is the radian frequency difference,

$$
\Delta \omega=\omega_{1}-\omega_{2}
$$

and $\sigma_{r}^{2}$ is the variance of $r(t)$. Thus, $\ddot{p}(t)$ is an unbiased estimate of $\Delta \omega$ when properly scaled by ${\sigma_{r}}^{-2}$.

Many data transmission modulation methods have a signal power spectrum symmetric about the carrier frequency, the most important examples being PSK and QAM [11]. The quadricorrelator is thus a suitable FD for carrier recovery with these modulation methods. For the particular case of biphase modulation, Cahn [8] has suggested a FD structure similar to the quadricorrelator, except that it includes an additional $I(d Q / d t)$ term. From the foregoing, it is evident that the simpler quadricorrelator would suffice. Bellisio [6] applied the quadricorrelator to baseband PAM timing recovery, exploiting the symmetry about the baud frequency of the pulse waveform spectrum generated by a NRZ data transition detector (differentiator followed by dead-zone quantizer).

Finally, we mention that many authors include limiters in both $I$ and $Q$ channels. This simplifies implementation of the correlation multiplier, which must have a very small offset to control static phase error, as well as insures a zero FD output after acquisition and eliminates the $\sigma_{r}{ }^{2}$ dependence in (3.2).

\subsection{Rotational Frequency Detector}

The rotational FD, in contrast to the quadricorrelator, is constructed of predominately digital circuitry and includes no filtering functions. Consequently, it is particularly well suited to integrated circuit implementation, but is also inherently limited to lower frequency operation than the quadricorrelator.

The rotational FD is simplest to describe for measurement of the frequency difference between two square waves, although it offers no particular advantage for that application over circuits described by Oberst [9] . That description is given in Section 3.3, and the simple generalizations to timing and

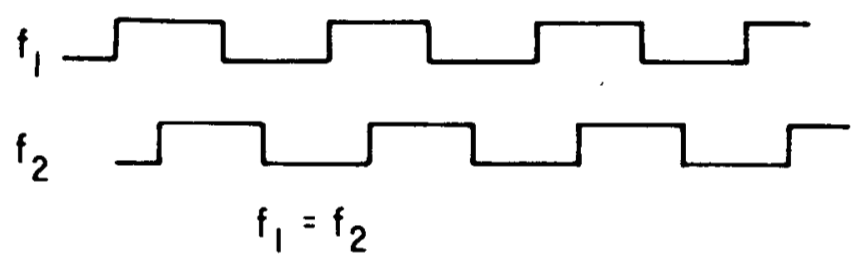

(a)

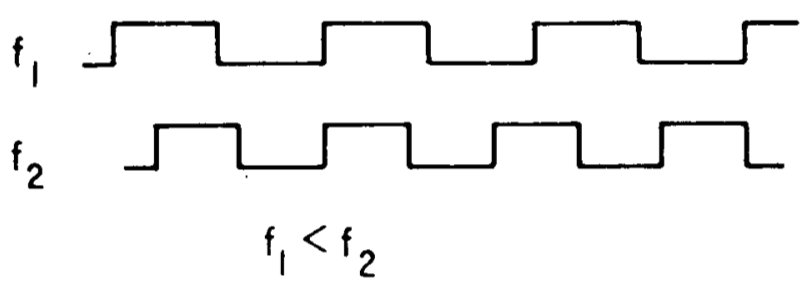

(b)

Fig. 5. Situations to be Distinguished by FD.

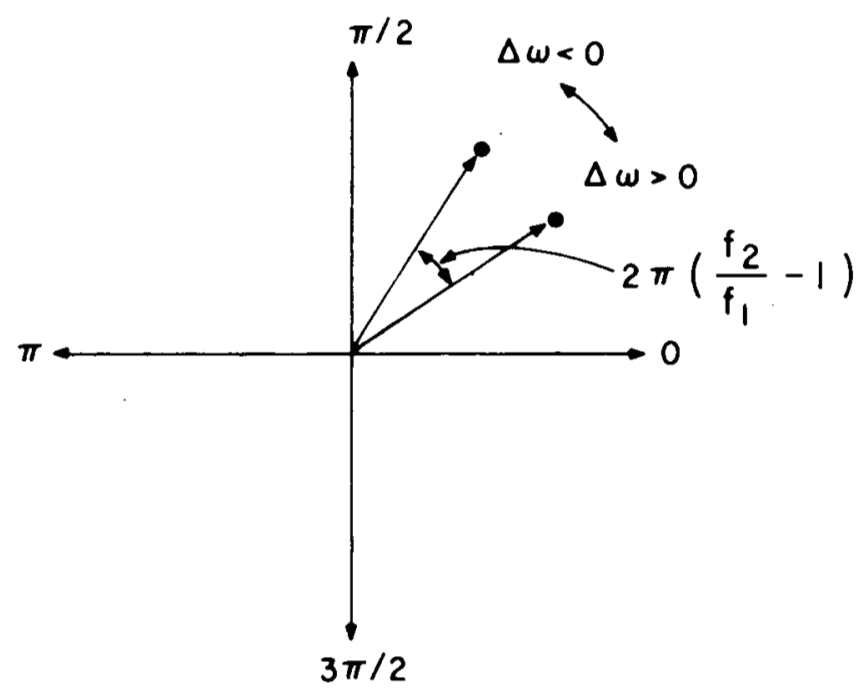

Fig. 6. Phasor Diagram of Two Successive Transitions of $f_{1}$ Relative to Phase of $f_{2}$.

carrier recovery are described in Section 3.4 and 3.5. The effect of noise and phase jitter is analyzed in Section 3.6.

\subsection{Two Square Waves}

Two of the three cases to be distinguished by the FD are shown in Figure 5. These cases would easily be recognized by a human observer watching the waveforms on an oscilloscope. When $f_{1}=f_{2}$, the transitions of $f_{1}$ maintain a fixed relationship to those of $f_{2}$. When $f_{1}<f_{2}$, the transitions of $f_{1}$ advance in phase relative to those of $f_{2}$, and vice versa when $f_{1}>f_{2}$. An excellent way to view the situation is to draw a phasor diagram as in Figure 6. One cycle ( $2 \pi$ radians) of $f_{2}$ is shown and the two phasors represent the relative phase of two successive transitions of $f_{1}$. The angle of rotation is readily shown to be $2 \pi\left(\left(f_{2} / f_{1}\right)-1\right)$, which is counterclockwise if $f_{1}<f_{2}$ and clockwise if $f_{1}>f_{2}$. Hence detecting the sign of the frequency difference is equivalent to determining the direction of rotation in Figure 6, while the magnitude of the frequency difference is related to the angle of rotation. 


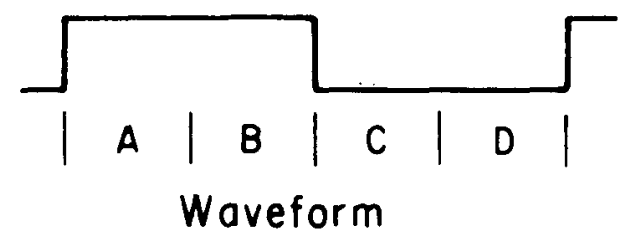

(a)

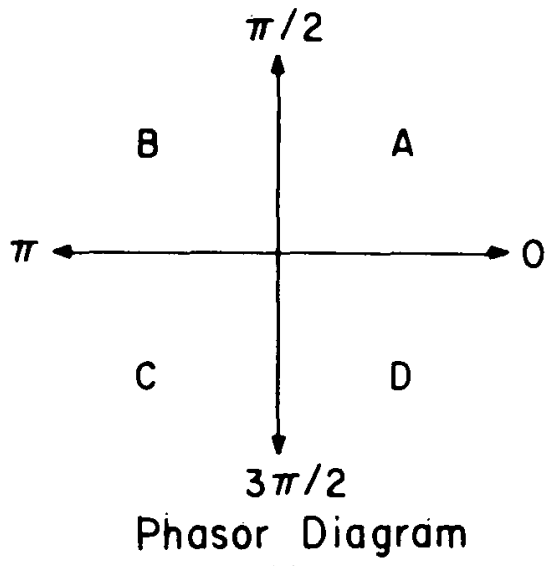

(b)

Fig. 7. Division of VCO Cycle into Four Quadrants.

A circuit which detects the direction of rotation can be built as follows: Assume $f_{2}$ is the VCO frequency, and divide each cycle into four quadrants labeled $A, B, C$, and $D$ as in Figures $7(\mathrm{a})$ and (b). This can be accomplished by actually running the $\mathrm{VCO}$ at four times frequency $f_{2}$, and dividing by four to obtain $f_{2}$ itself. Further assume that the PD is designed so that in-lock the PLL will maintain the positive transition of $f_{1}$ in the vicinity of the positive transitions of $f_{2}$ (other PD designs can be handled in like manner). Therefore, in-lock we would expect to observe positive tranisitions of $f_{1}$ predominately or exclusively in quadrants $A$ and $D$. To ensure that the FD will produce an output rarely if ever in-lock, it will operate only upon the observation of positive transitions of $f_{1}$ in quadrants $B$ and $C$.

Let the $k$ th cycle of $f_{2}$ be denoted by a $k$-subscript. The situation $f_{1}>f_{2}$ can be recognized by observation of $C_{k}$ followed by $B_{k+1}$, in which case the FD generates a positive pulse. Similarly, if a $B_{k}$ is followed by $C_{k+1}$, the FD generates a negative pulse, in recognition that $f_{1}<f_{\dot{2}}$.

The FD does not generate a pulse for every pair of $f_{1}$ transitions, since the rather special conditions of the last paragraph must be met. In particular, they will hopefully seldom be met in-lock, when no FD output is desired, since the PLL should serve to maintain the transition of $f_{1}$ in quadrants $A$ and $D$.

The FD is characterized by the mean value of the pulses at its output, since that mean value serves to charge or discharge the integrating capacitor in the loop filter. That mean value is, assuming FD positive and negative output pulses have equal area,

$$
\mu_{\mathrm{FD}}=\operatorname{Pr}\{\text { positive pulse }\}-\operatorname{Pr}\{\text { negative pulse }\}
$$

If the frequency difference is $\Delta \omega$; the angle of rotation is $-\Delta \omega / f_{1}$ radians, and $\mu_{F D}$ can readily be calculated by assum-

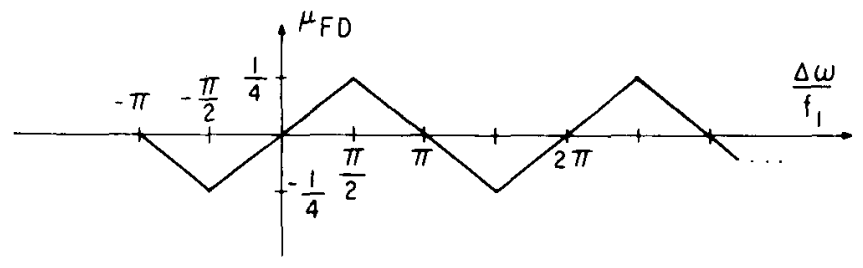

Fig. 8. FD Characteristic for Two Square Waves.

ing that the phasor in cycle $k$ is uniformly distributed from 0 to $2 \pi$ radians in Figure $7(\mathrm{~b})$. For example, if the angle of rotation of two successive phasors is $\phi<\pi / 2$, then an FD output is generated only when the first phasor is within an angle $\phi$ of the $\pi$-axis, an event which has probability $\phi / 2 \pi$. By a simple extension of this argument, the plot of $\mu_{\mathrm{FD}}$ of Figure 8 can be generated. The characteristic is periodic for $f_{1}>f_{2}$, since multiple cycles of $f_{1}$ in a period of $f_{2}$ cannot be distinguished from a single cycle by the FD circuit as described. It is not periodic for $f_{1}<f_{2}$ since, if the period of $f_{1}$ is too great, successive positive transition of $f_{1}$ will not occur within two periods of $f_{2}$ and the FD will generate no output.

As seen from Figure 8, the useful range of the FD is

$$
|\Delta \omega|<\pi f_{1}=\frac{\omega_{1}}{2}
$$

That is, a $50 \%$ offset in the initial VCO frequency $f_{2}$ can be tolerated. The range of linearity of the $\mathrm{FD}$, that is, the range over which the model of Figure 2(b) is accurate, is $|\Delta \omega| \leqslant$ $\omega_{1} / 4$. The largest FD output is at $\Delta \omega= \pm \omega_{1} / 4$, where the probability of an output pulse is 0.25 .

\subsection{Timing Recovery}

In timing recovery it is standard to generate a sequence of timing pulses from the data waveform. For example, Bellisio [6] describes a circuit consisting of a differentiator and deadzone rectifier which generates data transition pulses from an NRZ data waveform. The nominal spacing between two successive pulses can be any multiple of the baud interval $T=$ $1 / f_{1}$ since a pulse is only generated by a data transition. As in Section 3.3 we let $f_{2}$ be the VCO output frequency.

The FD described in Section 3.3 works for this case, where the quadrants of $f_{2}$ in which the data transition pulses occur are observed. The calculation of the FD output mean is similar, except that in addition to the requirement for two successive phasors to span the $\pi$-axis, there must be two data transitions in a row in order for an FD pulse to be generated. Thus, the FD characteristic of Figure 8 remains valid, except that $\mu_{F D}$ must be multiplied by the probability of two data transitions in a row ( 0.25 for equally likely independent data). The FD range is $50 \%$ of the baud rate, which is comparable to that reported by Bellisio [6] and more than adequate for the elimination of a VCXO.

\subsection{Carrier Recovery}

As mentioned in the introduction, the motivation for using an FD to aid acquisition in carrier recovery is somewhat dif- 


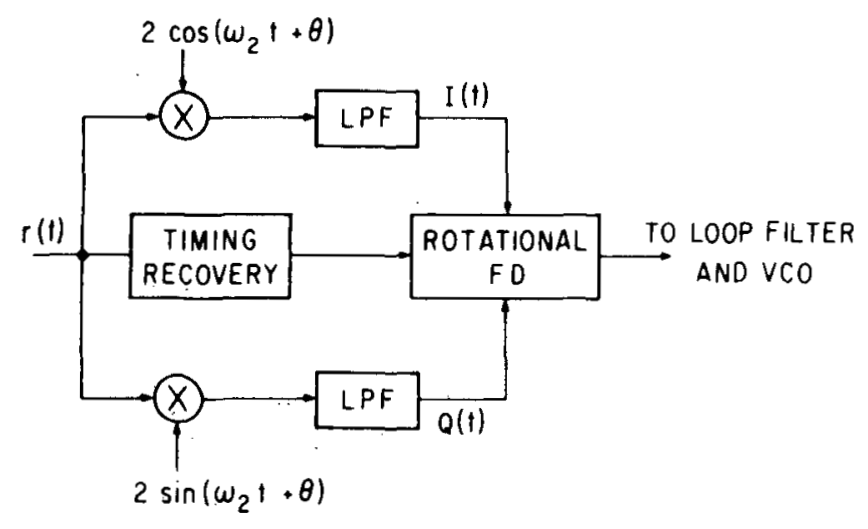

Fig. 9. Configuration of the Rotational FD in Carrier Recovery.

ferent than in timing recovery, for it is usually not practical to eliminate the VCXO due to the problem of false lock to a data sideband to be described shortly. Acquisition remains a problem, however, since worst-case frequency offsets can still exceed the desired loop bandwidth by many orders of magnitude. The problem is particularly acute in microwave radio transmission, where even very accurate RF oscillator frequencies can result in absolute frequency offsets of 50 to 100 $\mathrm{kHz}$, while carrier recovery loop bandwidths are more typically in the range of 0.1 to $1.0 \mathrm{kHz}$.

The signal used by the FD to extract carrier frequency offset is assumed to be of the form of (3.1), where $\omega_{1}$ would typically be the carrier frequency at IF. The object of the FD. is to estimate $\Delta \omega$ given by (3.3), where $\omega_{2}$ is the IF local oscillator frequency generated by the VCO in our carrier recovery PLL. The configuration of the FD is shown in Figure 9. The first step is to demodulate to baseband with quadrature carriers (at frequency $\omega_{2}$ ) just as for the Costas loop and quadricorrelator; the resulting quadrature baseband signals $I(t)$ and $Q(t)$ are given by (A.1). The FD operates on $I(t)$ and $Q(t)$, while timing recovery is performed on $r(t)$. The major requirement of the rotational $F D$ is that timing recovery acquisition occur before the FD output is valid and carrier recovery acquisition is initiated. The interesting property that timing recovery can be achieved independent of carrier recovery can be seen from squaring $r(t)$ in (3.1) and eliminating the double frequency term; the result is $\left(x_{c}{ }^{2}(t)+x_{s}{ }^{2}(t)\right) / 2$, which will have a baud frequency component suitable for extraction.

The first operation of the FD is to sample $I$ and $Q$ at the baud interval $k T$; from (A.1) the result is

$$
\begin{aligned}
I(k T) & =x_{c}(k T) \cos (k \Delta \omega T-\theta)-x_{s}(k T) \sin (k \Delta \omega T-\theta) \\
Q(k T) & =-x_{c}(k T) \sin (k \Delta \omega T-\theta)-x_{s}(k T) \cos (k \Delta \omega T-\theta) .
\end{aligned}
$$

Consider first the case $\Delta \omega=\theta=0$ following acquisition. The point $(I(k T), Q(k T))$ when plotted in a two-dimensional plane is, in fact, one of the data points in the two-dimensional signal constellation corresponding to the modulation method. We show two examples in Figure 10(a) and (b), two-level (biphase) and four-level (QPSK) phase-shift keying. Biphase serves as a basis of comparison to the work of Cahn [8], while

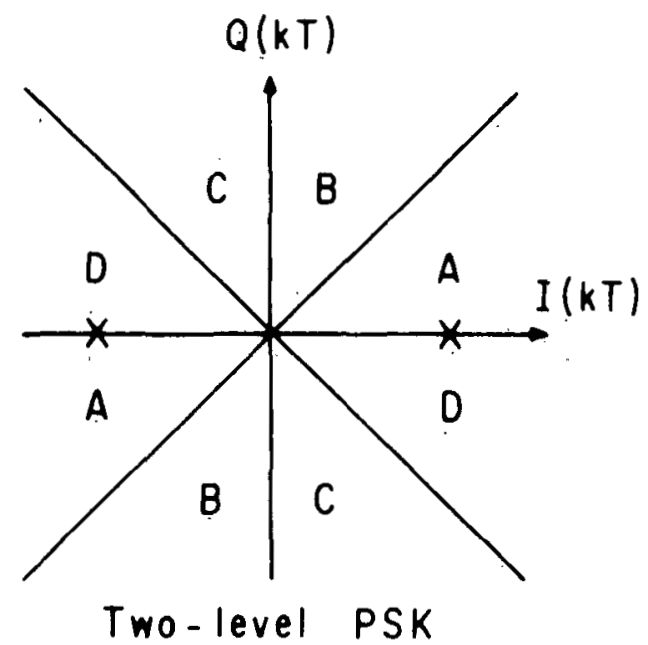

(a)

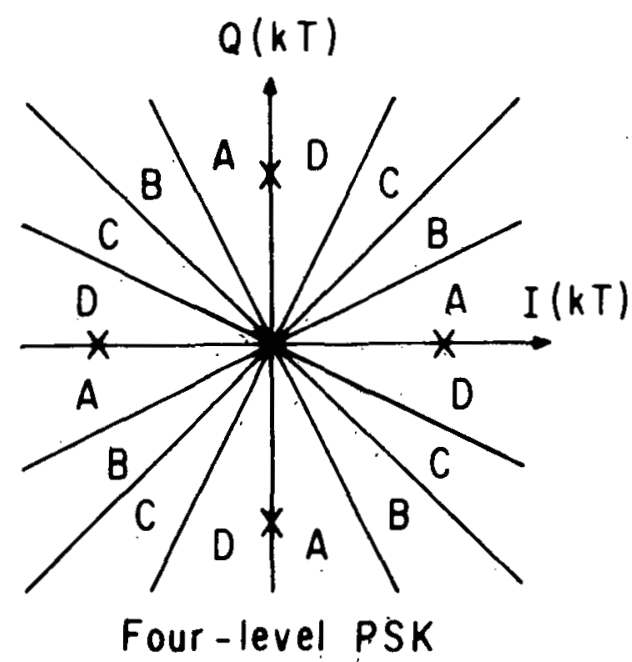

(b)

Fig. 10. Two-Dimensional Signal Constellations and Rotational FD Thresholds (Constellation shown for $\Delta \omega=\omega=0$ ).

QPSK demonstrates how the technique generalizes to more complicated constellations.***

When there îs a frequency difference, we recognize (3.6) as the parametric equations of a circle; that is, the signal constellation is rotated by an angle $k \Delta \omega T-\theta$. Rotation is clockwise if $\Delta \omega>0$. The nature of the problem of false lock to a data sideband is now clearly evident; in biphase modulation rotation by $\Delta \omega T$ equal to multiples of $\pi$ radians can clearly not be distinguished from $\Delta \omega T=0$. Thus, any FD characteristic must be periodic in $\Delta \omega=\pi / T$, and the maximum useful range of any FD is

$$
|\Delta \omega| \leqslant \pi / 2 T, \text { biphase. }
$$

Thus, the initial VCO frequency musst not deviate from the carrier frequency in magnitude by more than one-quarter the

\footnotetext{
*** It is also possible to restrict operation of the FD to a subset of the data points, if that subset can be unambiguously identified in the face of rotation. For exampie, in 16-level QAM, restriction to the four inner data points results in operation identical to QPSK.
} 
baud rate. For QPSK, (3.7) is replaced by

$$
|\Delta \omega| \leqslant \pi / 4 T, \text { QPSK }
$$

since false lock occurs when rotation is by $\pi / 2$ radians, and the maximum VCO offset is one-eighth the baud rate.

Shown on Figure 10, in addition to the signal constellation, are the radial thresholds required for the rotational FD. Some of these thresholds can also serve as slicers fot data decisions as well as for implementing a bang-bang type of PD. The radial thresholds divide the angle between each pair of adjacent data points into four quadrants $A, B, C, D$. In each baud interval the quadrant actually observed is independent of the data, and depends only on the angle of rotation $(k \Delta \omega T-\theta)$. As before, to insure infrequent $\mathrm{FD}$ pulses in lock when $\theta \cong 0$, operation is restricted to quadrants $B$ and $C$. The actual circumstances in which an FD pulse is generated are identical to the square wave and timing recovery cases.

The FD characteristic is plotted in Figure 11. The highest probability of a pulse output is 0.25 and óccurs at $|\Delta \omega T|=$ $\alpha / 2$, and the range of useful operation is $|\Delta \omega T| \leqslant \alpha$, where $\alpha=\pi / 2$ for biphase and $\alpha=\pi / 4$ for QPSK. Since these figures are consistent with (3.7) and (3.8) it follows that the rotational FD has as large a range of operation for carrier recovery as any FD.

\subsection{Effect of Phase Jitter}

The plots of $\mu_{\mathrm{FD}}$ presented thus far have not taken into account the effects of noise and intersymbol interference. Since the rotational FD is sensitive to the angle of rotation, which in turn is influenced by these factors, there is concern that they might significantly affect FD operation.

The situation is considered in Appendix B, where it is shown that if the phase jitter is small relative to $\pi / 2, \pi / 4$, or $\pi / 8$ for the timing recovery, biphase, or QPSK situations, respectively, the effect of phase jitter is virtually absent, this in spite of any statistical dependencies which may exist between successive samples of phase jitter. For phase jitter with amplitude less than twice the previously mentioned values, the effect is to change the shape of the FD characteristic (basically, round the corners), but not otherwise adversely affect its operation. Even larger phase jitter will have a significant adverse effect on FD operation, but is not likely to be encountered in practice, since the effect of this large jitter on error rate would also be substantial.

\subsection{EXPERIMENTAL RESULTS}

Experimental results on the use of the quadricorrelator in timing recovery were reported by Bellisio [6]. We report here on experimental results obtained in the implementation of a rotational FD in a carrier recovery application. The terrestrial microwave system to which jt was applied employed a 16-point signal constellation and $10 \mathrm{Mbit} / \mathrm{s}$ data rate. References [12-13] describe timing and carrier recovery techniques which are typical for this type of system.

This particular system protection switches at an error rate of $10^{-6}$, which corresponds to a baseband SNR of about 22 dB. Reliable acquisition was experimentally observed for an

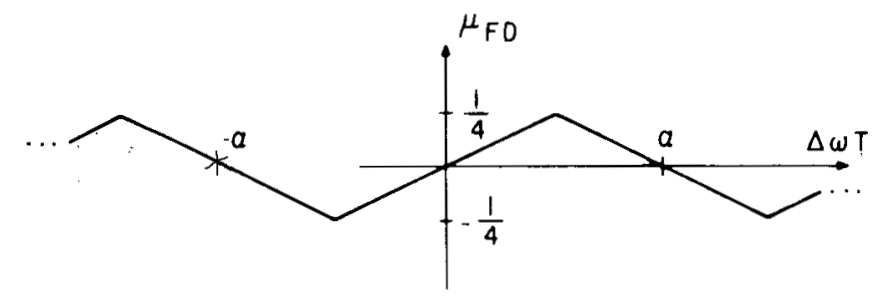

Fig. 11. FD Characteristic for Carrier Recovery.

SNR of $15 \mathrm{~dB}$ or less, which is substantially lower than necessary for this type of system. Figure 12 shows the VCO control line during several acquisitions at SNR's of 22,20 , and $15 \mathrm{~dB}$ starting from a worst-case carrier frequency offset $(133 \mathrm{kHz})$. The initial flat portion of the curves corresponds to the period of timing recovery acquisition, which must precede carrier acquisition for the rotational FD. Total acquisition time is about 15,25 , and $45 \mathrm{~ms}$ for the three cases.

\subsection{CONCLUSIONS}

The use of a FD to aid PLL timing and carrier acquisition is a very advantageous technique; the major impediment to its use appears to have been the lack of suitable FD circuits. We have described two such circuits, the quadricorrelator and rotational FD, both of which have a broad applicability. For timing recovery the rotational FD is somewhat simpler, particularly for integrated circuit implementation, since it is digital and requires no multipliers or filters; however, it is also limited to lower data rates. For carrier recovery there appears to be no great difference in the difficulty of implementation, since both circuits require quadrature mixers followed by circuitry which operates at approximately the baud rate. In some instances a substantial portion of the rotational FD circuitry can simultaneously serve other purposes (such as PD and data thresholding), in which case it becomes more attractive.

\section{APPENDIX A \\ QUADRICORRELATOR OUTPUT MEAN VALUE}

Let wide-sense stationary input signal $r(t)$ be written in the form of (3.1) and assume that the power spectrum of $r(t)$ is symmetrical about radian frequency $\omega_{1}$ so that $x_{c}(t)$ and $x_{s}(t)$ are uncorrelated. Assuming that $\omega_{1}+\omega_{2}$ terms are rejected by the low pass filters in Figure 4,

$$
\begin{aligned}
& I(t)=x_{c}(t) \cos (\Delta \omega t-\theta)-x_{s}(t) \sin (\Delta \omega t-\theta) \\
& Q(t)=-x_{c}(t) \sin (\Delta \omega t-\theta)-x_{s}(t) \cos (\Delta \omega t-\theta)
\end{aligned}
$$

where $\Delta \omega$ is given by (3.3). The crosscorrelation of $I(t)$ and $Q(t)$ is then easily shown to be, using the fact that $x_{c}(t)$ and $x_{s}(t)$ are uncorrelated,

$$
\begin{aligned}
R_{I Q}(\tau) & =E[I(t) Q(t+\tau)] \\
& =\frac{j}{4}\left(e^{j \Delta \omega \tau}-e^{-j \Delta \omega \tau}\right)\left(R_{c}(\tau)+R_{s}(\tau)\right)
\end{aligned}
$$




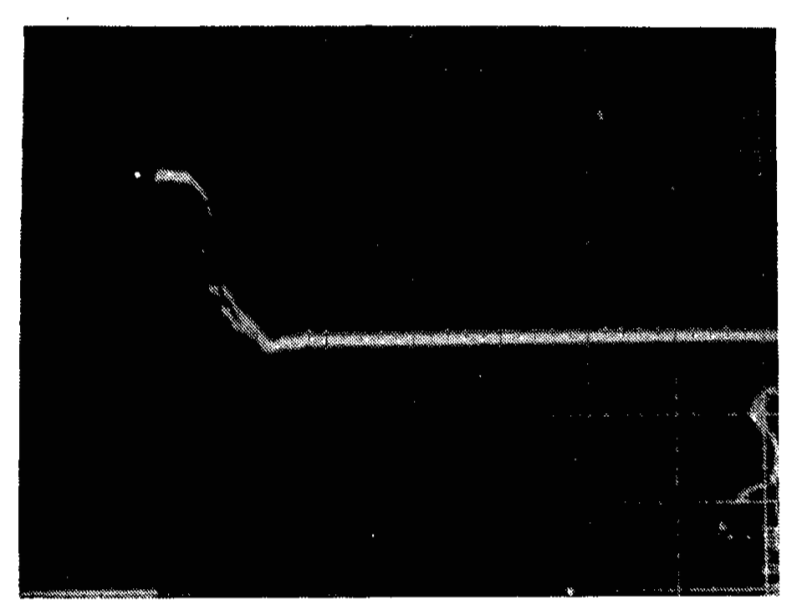

(a)

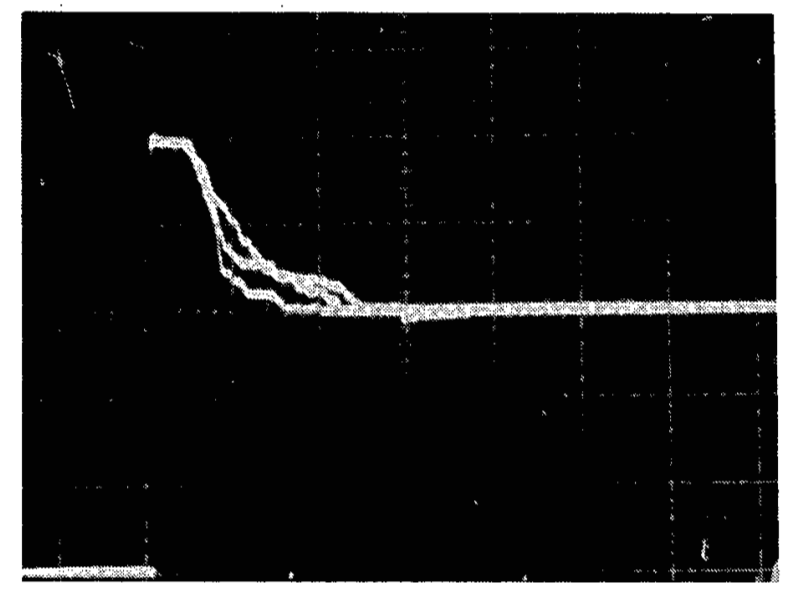

(b)

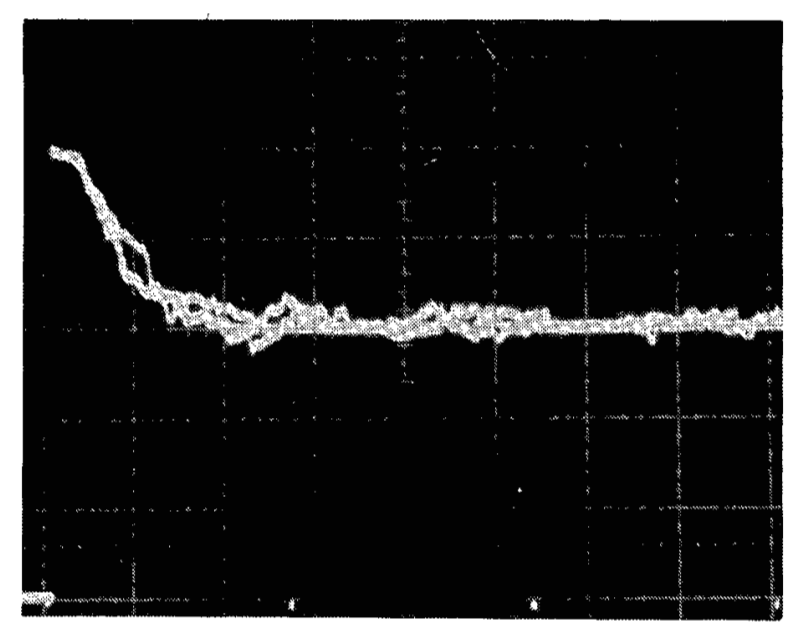

(c)

Fig. 12. Rotational FD Carrier Recovery Acquisition. (a) $S N R=22$ $\mathrm{dB}(10 \mathrm{~ms} /$ division $)$. (b) $\mathrm{SNR}=20 \mathrm{~dB}(10 \mathrm{~ms} /$ division $)$. (c) $\mathrm{SNR}=$ $15 \mathrm{~dB}(20 \mathrm{~ms} /$ division $)$. where $R_{c}(\tau)$ and $R_{s}(\tau)$ are the autocorrelation functions of $x_{c}(t)$ and $x_{s}(t)$. The cross power spectral density, the Fourier transform of (A.2), is

$$
\begin{aligned}
S_{I Q}(\omega)= & \frac{j}{4}\left\{S_{c}(\omega-\Delta \omega)-S_{c}(\omega+\Delta \omega)\right. \\
& \left.+S_{s}(\omega-\Delta \omega)-S_{s}(\omega+\Delta \omega)\right\} .
\end{aligned}
$$

The mean of $p(t)$ is thus

$$
\begin{aligned}
E p(t)= & E\left[Q(t) \frac{d I(t)}{d t}\right] \\
= & \int_{-\infty}^{\infty}(j \omega)^{*} S_{I Q}(\omega) \frac{d \omega}{2 \pi} \\
= & \frac{1}{4} \int_{-\infty}^{\infty} \omega\left\{\dot{S}_{c}(\omega-\Delta \omega)-S_{c}(\omega+\Delta \omega)\right. \\
& \left.+S_{s}(\omega-\Delta \omega)-S_{s}(\omega+\Delta \omega)\right\} \frac{d \omega}{2 \pi} .
\end{aligned}
$$

Changing variables in this integral, we obtain finally

$$
E p(t)=\frac{1}{2} \Delta \omega \int_{-\infty}^{\infty}\left(S_{c}(\omega)+S_{s}(\omega)\right) \frac{d \omega}{2 \pi}
$$

Recognizing that the power in $r(t)$ is one-half the sum of the powers in $x_{c}(t)$ and $x_{s}(t),(3.2)$ follows.

\section{APPENDIX B}

We can model the effect of phase jitter by assuming the angle of rotation at the $k$ th baud interval is $\left(k \Delta \omega T-\theta+\theta_{k}\right)$, where $\theta_{k}$ is a random phase jitter component. It is important that we not assume that $\theta_{k}$ and $\theta_{k+1}$ are independent, since intuitively dependencies should have a particularly strong influence. In order to recalculate $\mu_{F D}$ for this case, we let $\theta$ be uniformly distributed on $[0,2 \pi]$ as before. The starting angle is $\left(k \Delta \omega T-\theta+\theta_{k}\right)$, and the angle of rotation is $(\Delta \omega T+$ $\left.\theta_{k+1}-\theta_{k}\right)$. The key to simplifying the problem is to first condition on $\theta_{k}$ and $\theta_{k+1}$, and take the expectation over $\theta$, that expectation being the same as previously determined but with $\Delta \omega T$ replaced by $\left(\Delta \omega T+\theta_{k+1}-\theta_{k}\right)$. Thus, completing the expectation over $\theta_{k}$ and $\theta_{k+1}$,

$$
\mu_{\mathrm{FD}}=E\left[F\left(\Delta \omega T+\theta_{k+1}-\theta_{k}\right)\right]
$$

where $F(\Delta \omega T)$ is the FD characteristic of Figures 8 or 11 .

Equation (B.1) is exact, but for the special case where the argument of $F$ is in the linear region with high probability, where $F(\omega)=K \omega$,

$$
\begin{aligned}
\mu_{\text {F D }} & \cong E\left[K\left(\Delta \omega T+\theta_{k-1}-\theta_{k}\right)\right] \\
& =K\left(\Delta \omega T+E \theta_{k+1}-E \theta_{k}\right) \\
& =F(\Delta \omega T)
\end{aligned}
$$


where we have made the further assumption that $E \theta_{k+1}=$ $E \theta_{k}$ (not necessarily zero). The implication of (B.2) is that the phase jitter has had no effect whatsoever on $\mu_{\mathrm{FD}}$. Note that no assumption of independence of $\theta_{k}$ and $\theta_{k+1}$ has been made.

When this special case is violated, (B.1) can be used to estimate the effect. If $\theta_{k+1}-\theta_{k}$ has probability density $f(\cdot)$, then (B.1) becomes

$$
\mu_{\mathrm{FD}}=\int F(\Delta \omega T+\phi) f(\phi) d \Phi .
$$

Thus, if the argument $(\Delta \omega T+\phi)$ is not confined to the linear region of $F$, the effect is seen to be a smoothing of the corners of the FD characteristic. If $f(\phi)$ spans a significant portion of the period of $F$, then there is a significant deterioration of the FD operation.

\section{ACKNOWLEDGMENT}

The author is indebted to F. Stevens for the experimental results provided in Section 4.0 .

\section{REFERENCES}

1. F. M. Gardner, Phase-Lock Techniques, New York: Wiley, 1966.

2. D. Richman, "Color-Carrier Reference Phase Synchronization Accuracy in NTSC Color Television," Proc. IRE, Vol. 42, p. 106, January 1954.

3. T. B. Pickard, "The Effect of Noise on a Method of Frequency Measurement," IRE Trans. Information Thy, Vol. IT-4, p. 83, June 1958.
4. R. F. Pawula, "Analysis of an Estimator of the Center Frequency of a Power Spectrum," IEEE Trans. Information Thy, Vol. IT-14, p. 669, September 1968.

5. S. R. J. Axelsson, "Analysis of the Quantizing Error of a ZeroCounting Frequency Estimator," IEEE Trans. Information Thy, Vol. IT-22, P. 596, September 1976.

6. J. A. Bellisio, "New Phase-Locked Timing Recovery Method for Digital Regenerators," Int. Conf. Communications Record, Philadelphia, pp. 10-17, June 1976.

7. J. H. Park, Jr., "An FM Detector for Low S/N," IEEE Trans. Comm., Vol. COM-18, p. 110, April 1970.

8. C. R. Cahn, "Improving Frequency Acquisition of a Costas Loop," IEEE Trans. on Comm., Vol. COM-25, p. 1453, December 1977.

9. J. F. Oberst, "Generalized Phase Comparators for Improved PLL Acquisition," IEEE Trans. on Comm. Tech., Vol. COM-19, p. 1142, December 1971.

10. P. K. Runge, "Phase Locked Loops with Signal Injection for Increased Pull-In Range and Reduced Output Phase Jitter," IEEE Trans. Comm., Vol. COM-24, p. 636, June 1976.

11. R. W. Lucky, J. Salz, E. J. Weldon, Jr., Principles of Data Communication, New York, McGraw-Hill, 1968.

12. C. W. Anderson, S. G. Barber, "Modulation Considerations for a $91 \mathrm{Mb} / \mathrm{s}$ Digital Radio," IEEE Trans. on Communications, Vol. COM-26, May 1978, p. 523.

13. C. R. Hogge, "Carrier and Clock Recovery for 8 PSK Synchronous Demodulation," IEEE Trans. on Communications, Vol. COM-26, May 1978, p. 528.

14. R. Citta, "Frequency and Phase Lock Loop," IEEE Trans. Consumer Electronics, Vol. CE-23, Aug. 1977, p. 358.

David G. Messerschmitt (S'65-M'68-SM'78), for a photograph and biography please see page 1027 of the July 1979 issue of this TRANSACTIONS. 